\title{
EXTENSION OF VIKOR METHOD FOR MCDM UNDER BIPOLAR FUZZY SET
}

\section{BASHAYER ALSOLAME* AND NOURA OMAIR ALSHEHRI}

\author{
Department of Mathematics, Faculty of Science, University of Jeddah
}

*Corresponding author: baalsolame@uj.edu.sa

\begin{abstract}
In this paper, we extend the VIKOR method (Serbian name: VlseKriterijumska Optimizacija I Kompromisno Resenje, means multi-criteria optimization and compromise solution) to solve multi-criteria decision making (MCDM) with bipolar fuzzy environment. Firstly, the bipolar fuzzy set concept is described. Then, proposed the VIKOR strategy in bipolar set environment to handle MCDM problems. Finally, two numerical examples illustrate an application of BF-VIKOR method, and analysis the results of different values of the decision making weights of criteria on ranking order of the alternatives.
\end{abstract}

\section{INTRODUCTION}

Multi-Criteria Decision Making (MCDM) has seen an incredible amount of use over last several decades. Its role in different applications areas has increased significantly, especially as new methods develop and as old methods improve. In classical MCDM methods, the goal is to designate a preferred alternative, classify alternatives in a small number of categories and rank alternatives in a subjective preference order. However, most of the real world decisions are made in an environment in which goals and constraints cannot be precisely expressed due to their complexity. The fuzzy sets theory was first proposed by Zadeh in 1965 ( [1], [2]). Afterward, Bellman and Zadeh described a decision-making method in a fuzzy environment [3]. Multi-criteria optimization and compromise solution (VIKOR in Serbian) method, developed by Opricovic (1998) [4], Opricovic and Tzeng (2004) [5], is one of the most outranking MCDM method. The first paper that proposes to use fuzzy inputs with VIKOR method was published in 2002 [6]. Later, Opricovic [7] proposes a

Received August 15 ${ }^{\text {th }}, 2020$; accepted September $7^{\text {th }}, 2020$; published September $29^{\text {th }}, 2020$.

2010 Mathematics Subject Classification. 03E72.

Key words and phrases. bipolar fuzzy set; VIKOR method.

(C)2020 Authors retain the copyrights of their papers, and all open access articles are distributed under the terms of the Creative Commons Attribution License. 
fuzzy extension of VIKOR to find a fuzzy compromise solution. Wang and Chang [8] introduced fuzzy VIKOR for solving multi-criteria group decision-making problem. Since that, many researchers have been dealing with decision-making problems by applying VIKOR method in fuzzy environment ( [9], [10], [11], [12], [13]), interval-valued intuitionistic fuzzy environment [14], intuitionistic fuzzy VIKOR [15] and hesitant fuzzy VIKOR [16].

In 1994, Zhang [17] proposed the concept of bipolar fuzzy sets, which is a generalization of fuzzy set allowing the membership degree having two values. One locates in the interval $[0,1]$ represents the satisfaction degree of a certain property associated to the fuzzy set and the other locates in the interval $[-1,0]$ and represents the satisfaction degree of a counter-property to the concerned fuzzy set. Bipolarity is important to distinguish between positive information, which represents what is guaranteed to be possible, for example what has already been observed or experienced and negative information, which represents what is impossible or forbidden, or surely false. Thus providing an efficient approach to solving a widely larger number of complex decision making problems. In recent years, many decision-making problems have been applied in bipolar fuzzy environment. In this paper, we present bipolar fuzzy VIKOR (BF-VIKOR) method solving MCDM problems that are equipped with bipolar fuzzy information. We illustrate our proposed method with numerical examples.

\section{Preliminaries}

In this section, some preliminaries from the fuzzy set theory and bipolar fuzzy sets are induced.

Definition 2.1. A fuzzy set of the universe $X$ is a mathematical object $A$ described by its characteristic function (membership function) $\mu_{A}: X \rightarrow[0,1]$ defined as [1]:

$$
A=\left\{\left(x, \mu_{A}(x)\right) \mid x \in X\right\}
$$

Definition 2.2. A bipolar fuzzy set $A$ on the universe $X$ is defined as [17]:

$$
A=\left\{\left(x, \mu_{A}(x), \nu_{A}(x)\right) \mid x \in X\right\}
$$

where $\mu_{A}: X \rightarrow[0,1]$ is a positive membership degree denotes the satisfaction degree of an element to the property corresponding to $A$, and $\nu_{A}: X \rightarrow[-1,0]$ is the negative membership degree denotes the satisfaction degree of an element to some implicit counter-property corresponding to $A$.

\section{VIKOR METHOD}

The VIKOR method focuses on ranking and selecting from a set of alternatives with conflicting criteria, and determines compromise solution based on particular measure. Assuming that each alternative is 
evaluated according to each criterion function, the compromise ranking can be presented by comparing the degree of closeness to the ideal alternative. The compromise solution, whose foundation was established by $\mathrm{Yu}$ [18] and Zeleny [19], is a feasible solution, which is the closest to the ideal, and here "compromise" means an agreement established by mutual concessions. The multi-criteria measure for compromise ranking is developed from the $L_{p}$ - metric used as an aggregating function in a compromise programming method defined as:

$$
L_{p, i}=\left[\sum_{j=1}^{n}\left[w_{j} \frac{\left(f_{j}^{*}-f_{i j}\right)}{\left(f_{j}^{*}-f_{j}^{-}\right)}\right]^{p}\right]^{\frac{1}{p}}, 1 \leq p \leq \infty
$$

where $L_{1, i}$ is defined as the maximum group utility, and $L_{\infty, i}$ is defined as the minimum individual regret of the opponent.

For a MCDM problem involving $m$ alternatives and $n$ criteria, whereby the performances of the alternatives are expressed by using bipolar fuzzy set, Let

$\psi=\left\{\psi_{1}, \psi_{2}, \ldots, \psi_{m}\right\}=\left\{\psi_{i}: i=1,2, \ldots, m\right\}$ and $C=\left\{C_{1}, C_{2}, \ldots, C_{n}\right\}=\left\{C_{j}: j=1,2, \ldots, n\right\}$ be the sets of alternatives and criteria that are determined by a decision maker, respectively.

Formally, a bipolar fuzzy MCDM problem can be express in matrices format as follows:

$$
D=\left[\begin{array}{cccc}
\left(\mu_{11}, \nu_{11}\right) & \left(\mu_{12}, \nu_{12}\right) & \ldots & \left(\mu_{1 n}, \nu_{1 n}\right) \\
\left(\mu_{21}, \nu_{21}\right) & \left(\mu_{22}, \nu_{22}\right) & \ldots & \left(\mu_{2 n}, \nu_{2 n}\right) \\
\cdot & \cdot & \cdot & \cdot \\
\left(\mu_{m 1}, \nu_{m 1}\right) & \left(\mu_{m 2}, \nu_{m 2}\right) & \ldots & \left(\mu_{m n}, \nu_{m n}\right)
\end{array}\right]_{m \times n}
$$

The ratings of any alternative $\psi_{i} \in \psi$ for each criterion $C_{j}$ are given by a bipolar fuzzy set $F_{i}=\left\{\left(\psi_{i}, f_{i j}\right) \mid j=1,2, \ldots, n\right\}$ where $f_{i j}=\left(\mu_{i j}, \nu_{i j}\right)$ represent respectively, the satisfaction degree $\left(\mu_{i j} \in[0,1]\right)$ and the dissatisfaction degree $\left(\nu_{i j} \in[-1,0]\right)$ that are determined for $\psi_{i}$ with respect to $C_{j}$. Now, determine the best $f_{j}^{*}$ and the worst $f_{j}^{-}$value of all criterion functions, $j=1,2, \ldots, n$ as:

$$
\begin{aligned}
& f_{j}^{*}=\left\{\begin{array}{c}
\left(\max \mu_{i j}, \min \nu_{i j}\right), \text { for the benefit criterion } \\
\left(\min \mu_{i j}, \max \nu_{i j}\right), \text { for the cost criterion }
\end{array}\right\}, i=1,2, \ldots, m . \\
& f_{j}^{-}=\left\{\begin{array}{c}
\left(\min \mu_{i j}, \max \nu_{i j}\right), \text { for the benefit criterion } \\
\left(\max \mu_{i j}, \min \nu_{i j}\right), \text { for the cost criterion }
\end{array}\right\}, i=1,2, \ldots, m
\end{aligned}
$$

In the VIKOR development methodology $S_{i}$ (as $L_{1, i}$ in Equation 3.1) and $R_{i}$ (as $L_{\infty, i}$ in Equation 3.1) are used to formulate ranking measure [4], [5]. In light of the distance measure for each alternatives functions 
$f_{i j}=\left(\mu_{i j}, \nu_{i j}\right)$ and the best $f_{J}^{*}$ and the worst $f_{j}^{-}$value of all criterion functions.

$$
\begin{gathered}
S_{i}=\sum_{j=1}^{n} w_{j} \frac{d\left(f_{j}^{*}, f_{i j}\right)}{d\left(f_{j}^{*}, f_{j}^{-}\right)}, \\
R_{i}=\max _{j}\left[w_{j} \frac{d\left(f_{j}^{*}, f_{i j}\right)}{d\left(f_{j}^{*}, f_{j}^{-}\right)}\right]
\end{gathered}
$$

where $w_{j}$ are the weights of criteria, expressing the decision-makers preference of the criteria, such that $w_{j} \in[0,1]$ and $\sum_{j=1}^{n} w_{j}=1$.

Furthermore, the relative closeness degree of each alternative $\psi_{i}(i=1,2, \ldots, m)$ with respect to $S_{i}$ and $R_{i}$ is given as:

$$
Q_{i}=\frac{v\left(S_{i}-S^{*}\right)}{\left(S^{-}-S^{*}\right)}+\frac{(1-v)\left(R_{i}-R^{*}\right)}{\left(R^{-}-R^{*}\right)}
$$

where,

$$
\begin{aligned}
S^{*}=\min _{i} S_{i} & , \quad S^{-}=\max _{i} S_{i} \\
R^{*}=\min _{i} R_{i} & , \quad R^{-}=\max _{i} R_{i}
\end{aligned}
$$

$v$ and $(1-v)$ are the weights for the strategy of maximum group utility and individual regret, respectively.

Usually, the value of $v$ can be assumed to be $(v=0.5)$. The $Q_{i}$ represent the distance of $\psi_{i}$ alternative from the best solution "compromise solution". So the alternative, which has the minimum value in $Q_{i}$ would be the compromise solution if the following two conditions are satisfied:

C1) Acceptable advantage:

$$
Q\left(\psi^{(2)}\right)-Q\left(\psi^{(1)}\right) \geq \frac{1}{m-1}
$$

where, $\psi^{(1)}$ and $\psi^{(2)}$ are the top two alternatives in $Q_{i}$ [such that $Q_{i}$ sort in ascending order], $m$ is the number of the alternatives.

C2) Acceptable stability: the compromise solution should be the best rank by $S_{i}$ and/or $R_{i}$. If one of the conditions is not satisfied, then a set of compromise solutions is proposed, which consists of:

- $\psi^{(1)}$ and $\psi^{(2)}$ if only the condition $\mathrm{C} 2$ is not satisfied, or

- $\psi^{(1)}, \psi^{(2)}, \ldots, \psi^{(M)}$ if the condition $\mathrm{C} 1$ is not satisfied (Equation 3.8$) ; \psi^{(M)}$ is determined by the relation:

$$
Q\left(\psi^{(M)}\right)-Q\left(\psi^{(1)}\right)<\frac{1}{m-1} ; \text { maximum } M
$$




\section{VIKOR Algorithm}

According to the above discussion, the procedures of BF-VIKOR method can be summarized in Figure 1.

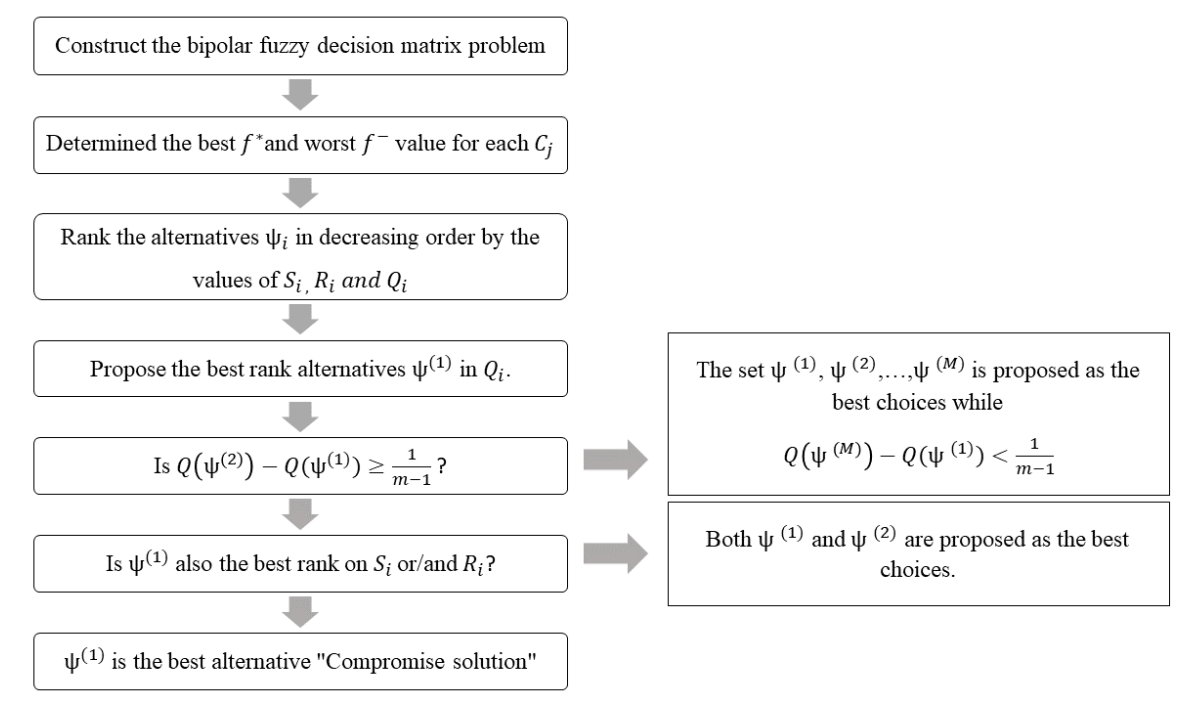

Figure 1. The Flow Chart of BF-VIKOR Algorithm

\section{Numerical Examples}

5.1. Example 1. Assume that we have a decision maker who is confused in choosing the a new location to start his sales business. After the initial consideration of the available alternatives, four alternatives has been identified as suitable. Suppose that he is evaluating suitable alternatives based on the following criteria:

$C_{1}$ :Rental space quality;

$C_{2}$ :Rental space adequacy;

$C_{3}$ :Location quality;

$C_{4}$ :Location distance from the city center, and

$C_{5}$ :Rental price. So, these locations represent the alternatives $\left\{\psi_{1}, \psi_{2}, \psi_{3}, \psi_{4}\right\}$ and the mentioned features represent the criteria $\left\{C_{1}, C_{2}, C_{3}, C_{4}, C_{5}\right\}$ in our MCDM problem. Ratings of the alternatives, in Table 1 , and weights of the criteria are given by the decision maker in matrices format with bipolar fuzzy and fuzzy values, respectively, as follows:

$W=\left[\begin{array}{lllll}0.18 & 0.24 & 0.21 & 0.16 & 0.21\end{array}\right]^{T}$ Clearly, the given weights satisfy the normalized condition.

From Equation 3.3, Equation 3.4 the best and worst values of all criterion ratings are determined as follows in Table 2. Further, the Equations 3.5 - 3.7 calculated the values of $S_{i}, R_{i}$ and $Q_{i}$ in Table 3 were $(v=0.5)$ and rank the alternatives by ranking $S_{i}, R_{i}$ and $Q_{i}$ in decreasing order as shown in Table 4 . Thus, the compromise solution is $\psi_{4}$ and $\psi_{1}$. 
TABLE 1. Ratings of the alternatives

\begin{tabular}{cccccc}
\hline & $C_{1}$ & $C_{2}$ & $C_{3}$ & $C_{4}$ & $C_{5}$ \\
\hline$\psi_{1}$ & $(0.67,-0.16)$ & $(0.79,-0.32)$ & $(1,0)$ & $(1,0)$ & $(0.81,-0.10)$ \\
\hline$\psi_{2}$ & $(0.47,-0.18)$ & $(0.43,-0.42)$ & $(1,-.26)$ & $(1,0)$ & $(0.77,-0.10)$ \\
\hline$\psi_{3}$ & $(0.64,-0.10)$ & $(0.61,-0.16)$ & $(0.49,0)$ & $(0.41,-0.10)$ & $(0.70,-0.13)$ \\
\hline$\psi_{4}$ & $(0.81,-0.10)$ & $(1,-0.15)$ & $(0.53,0)$ & $(0.53,-0.10)$ & $(0.60,-0.10)$ \\
\hline
\end{tabular}

TABLE 2. The best and worst value of all criterion functions

\begin{tabular}{ccc}
\hline & $f_{j}^{*}$ & $f_{j}^{-}$ \\
\hline$C_{1}$ & $(0.81,-0.18)$ & $(0.47,-0.10)$ \\
\hline$C_{2}$ & $(1,-0.42)$ & $(0.43,-0.15)$ \\
\hline$C_{3}$ & $(1,-0.26)$ & $(0.49,0)$ \\
\hline$C_{4}$ & $(1,-0.10)$ & $(0.41,0)$ \\
\hline$C_{5}$ & $(0.60,-0.10)$ & $(0.80,-0.13)$ \\
\hline
\end{tabular}

TABLE 3 . The values of $S_{i}, R_{i}$ and $Q_{i}$ for all alternatives

\begin{tabular}{ccccc}
\hline & $\psi_{1}$ & $\psi_{2}$ & $\psi_{3}$ & $\psi_{4}$ \\
\hline$S_{i}$ & 0.491 & 0.595 & 0.751 & 0.467 \\
\hline$R_{i}$ & 0.208 & 0.217 & 0.21 & 0.197 \\
\hline$Q_{i}$ & 0.311 & 0.726 & 0.826 & 0 \\
\hline
\end{tabular}

TABLE 4. Final ranking of alternatives

\begin{tabular}{cccccc}
\hline & $\psi_{1}$ & $\psi_{2}$ & $\psi_{3}$ & $\psi_{4}$ & Ranking \\
\hline$S_{i}$ & 0.491 & 0.595 & 0.751 & 0.467 & $\psi_{4}, \psi_{1}, \psi_{2}, \psi_{3}$ \\
\hline$R_{i}$ & 0.208 & 0.217 & 0.21 & 0.197 & $\psi_{4}, \psi_{1}, \psi_{3}, \psi_{2}$ \\
\hline$Q_{i}$ & 0.311 & 0.726 & 0.826 & 0 & $\psi_{4}, \psi_{1}, \psi_{2}, \psi_{3}$ \\
\hline
\end{tabular}

5.2. Example 2. Assume that we have a decision maker who is confused in choosing the best airline. After the initial consideration of the available alternatives, five alternatives has been identified as suitable. Suppose that he is evaluating suitable alternatives based on the following criteria:

$C_{1}$ :Empathy, represents how airline deal with the customer complaints and provide thoughtful services; $C_{2}$ :Assurance, represents the certainty that airline provides for customers;

$C_{3}$ :Tangibility, means the physical service presentation such as quality of the food, and $C_{4}$ :Cost, represents the tickets and services prices. 
So, these airlines represent the alternatives $\left\{\psi_{1}, \psi_{2}, \psi_{3}, \psi_{4}, \psi_{5}\right\}$ and the mentioned features represent the criteria $\left\{C_{1}, C_{2}, C_{3}, C_{4}\right\}$ in our MCDM problem. Ratings of the alternatives, in Table 5 , and weights of the criteria are given by the decision maker in matrices format with bipolar fuzzy and fuzzy values, respectively, as follows:

$$
W=\left[\begin{array}{llll}
0.25 & 0.3 & 0.25 & 0.2
\end{array}\right]^{T}
$$

Clearly, the given weights satisfy the normalized condition. Using the Equations 3.5 - 3.7 the values of $S_{i}$, $R_{i}$ and $Q_{i}$ are calculated as in Table 6.

TABLE 5. Rating of the alternatives

\begin{tabular}{ccccc}
\hline & $C_{1}$ & $C_{2}$ & $C_{3}$ & $C_{4}$ \\
\hline$\psi_{1}$ & $(0.5,-0.25)$ & $(0.8,-0.7)$ & $(0.3,-0.1)$ & $(0.6,-0.6)$ \\
\hline$\psi_{2}$ & $(0.2,-0.8)$ & $(0.9,-0.4)$ & $(0.6,-0.3)$ & $(0.55,-0.5)$ \\
\hline$\psi_{3}$ & $(0.33,-0.25)$ & $(0.75,-0.4)$ & $(0.25,-0.7)$ & $(0.3,-0.1)$ \\
\hline$\psi_{4}$ & $(0.65,-0.6)$ & $(0.3,-0.75)$ & $(0.8,-0.35)$ & $(0.65,-0.7)$ \\
\hline$\psi_{5}$ & $(1,-0.5)$ & $(0.4,-0.35)$ & $(0.2,-0.6)$ & $(0.25,-0.65)$ \\
\hline
\end{tabular}

TABLE 6 . The values of $S_{i}, R_{i}$ and $Q_{i}$ for all alternatives

\begin{tabular}{cccccccc}
\hline & $\psi_{1}$ & $\psi_{2}$ & $\psi_{3}$ & $\psi_{4}$ & $\psi_{5}$ & Ranking & Compromise solutions \\
\hline$S_{i}$ & 0.637 & 0.622 & 0.558 & 0.657 & 0.675 & $\psi_{3}, \psi_{2}, \psi_{1}, \psi_{4}, \psi_{5}$ & $\psi_{3}$ \\
\hline$R_{i}$ & 0.23 & 0.206 & 0.223 & 0.25 & 0.266 & $\psi_{2}, \psi_{3}, \psi_{1}, \psi_{4}, \psi_{5}$ & $\psi_{2}$ \\
\hline$Q_{i}(v=0.5)$ & 0.538 & 0.274 & 0.143 & 0.781 & 1 & $\psi_{3}, \psi_{2}, \psi_{1}, \psi_{4}, \psi_{5}$ & $\psi_{3}, \psi_{2}$ \\
\hline
\end{tabular}

After rank the alternatives by sorting the values $S_{i}, R_{i}$ and $Q_{i}$ in decreasing order. The results are three ranking lists, which is depicted in Table 6. So, the compromise solution are $\psi_{3}$ and $\psi_{2}$.

The parameter $v$ in VIKOR technique normally considered as $(v=0.5)$. Therefore, by changing value of $v$ in the interval $[0,1]$ is performed for the obtained results. The ranking for five alternatives under different $v$ values are illustrated in Table 7 . As can be seen, when $v$ is changed, there are some deviations in ranking of alternatives. $\psi_{3}$ is the best ranked alternative for $v \geq 0.7$; also, $\psi_{2}$ has the best rank for $v=0$. Moreover, $\psi_{5}$ is the worst ranked alternative for different values of $v$.

\section{Conclusion}

In MCDM methods the alternatives are compared against each other based on how they perform relative to each criterion. One the other hand, VIKOR method require comparison of the criteria to determine the relative importance of each criterion. The alternative with the highest rank is selected as the best compromise 
TABLE 7. Ranking orders of alternatives under different $v$ values.

\begin{tabular}{|c|c|c|c|c|c|c|c|c|}
\hline & & $\psi_{1}$ & $\psi_{2}$ & $\psi_{3}$ & $\psi_{4}$ & $\psi_{5}$ & Ranking & Compromise solutions \\
\hline$S_{i}$ & & 0.637 & 0.622 & 0.558 & 0.657 & 0.675 & $\psi_{3}, \psi_{2}, \psi_{1}, \psi_{4}, \psi_{5}$ & $\psi_{3}$ \\
\hline$R_{i}$ & & 0.23 & 0.206 & 0.223 & 0.25 & 0.266 & $\psi_{2}, \psi_{3}, \psi_{1}, \psi_{4}, \psi_{5}$ & $\psi_{2}$ \\
\hline \multirow[t]{11}{*}{$Q_{i}(v)$} & 0 & 0.399 & 0 & 0.285 & 0.722 & 1 & $\psi_{2}, \psi_{3}, \psi_{1}, \psi_{4}, \psi_{5}$ & $\psi_{2}$ \\
\hline & 0.1 & 0.427 & 0.055 & 0.257 & 0.734 & 1 & $\psi_{2}, \psi_{3}, \psi_{1}, \psi_{4}, \psi_{5}$ & $\psi_{2}, \psi_{3}$ \\
\hline & 0.2 & 0.455 & 0.109 & 0.228 & 0.746 & 1 & $\psi_{2}, \psi_{3}, \psi_{1}, \psi_{4}, \psi_{5}$ & $\psi_{2}, \psi_{3}$ \\
\hline & 0.3 & 0.482 & 0.164 & 0.2 & 0.758 & 1 & $\psi_{2}, \psi_{3}, \psi_{1}, \psi_{4}, \psi_{5}$ & $\psi_{2}, \psi_{3}$ \\
\hline & 0.4 & 0.51 & 0.219 & 0.171 & 0.769 & 1 & $\psi_{3}, \psi_{2}, \psi_{1}, \psi_{4}, \psi_{5}$ & $\psi_{3}, \psi_{2}$ \\
\hline & 0.5 & 0.538 & 0.274 & 0.143 & 0.781 & 1 & $\psi_{3}, \psi_{2}, \psi_{1}, \psi_{4}, \psi_{5}$ & $\psi_{3}, \psi_{2}$ \\
\hline & 0.6 & 0.566 & 0.328 & 0.114 & 0.793 & 1 & $\psi_{3}, \psi_{2}, \psi_{1}, \psi_{4}, \psi_{5}$ & $\psi_{3}, \psi_{2}$ \\
\hline & 0.7 & 0.594 & 0.383 & 0.086 & 0.805 & 1 & $\psi_{3}, \psi_{2}, \psi_{1}, \psi_{4}, \psi_{5}$ & $\psi_{3}$ \\
\hline & 0.8 & 0.621 & 0.438 & 0.057 & 0.816 & 1 & $\psi_{3}, \psi_{2}, \psi_{1}, \psi_{4}, \psi_{5}$ & $\psi_{3}$ \\
\hline & 0.9 & 0.649 & 0.493 & 0.029 & 0.828 & 1 & $\psi_{3}, \psi_{2}, \psi_{1}, \psi_{4}, \psi_{5}$ & $\psi_{3}$ \\
\hline & 1 & 0.677 & 0.547 & 0 & 0.84 & 1 & $\psi_{3}, \psi_{2}, \psi_{1}, \psi_{4}, \psi_{5}$ & $\psi_{3}$ \\
\hline
\end{tabular}

solution. In this paper, we proposed BF-VIKOR method Since the VIKOR method is an effective MCDM method to reach a compromise solution, particularly in a situation where the decision maker is not able to express his preference at the beginning. Furthermore, BFSs are an effective tool to depict fuzziness and bipolarity in assessment information. The obtained solution is compromised by a maximum group utility of the majority, and a minimum of the individual regret. In future study, we may apply VIKOR method to problems that are under two fuzzy concept(such that bipolar hesitant fuzzy).

Conflicts of Interest: The author(s) declare that there are no conflicts of interest regarding the publication of this paper.

\section{REFERENCES}

[1] L. Zadeh, Fuzzy Sets, Information and Control. 8 (3) (1965), 338-353.

[2] L. Zadeh, The concept of a linguistic variable and its application to approximate reasoning, Inform. Sci. 8 (3) (1975), 199-249.

[3] R. E. Bellman, L. A. Zadeh, Decision making in a fuzzy environment, Manage. Sci. 17 (4) (1970), 141-164.

[4] S. Opricovic, G.H. Tzeng, Compromise solution by MCDM methods: A comparative analysis of VIKOR and TOPSIS, Eur. J. Oper. Res. 156 (2) (2004), 445-455. 
[5] S. Opricovic, G.H. Tzeng, Extended VIKOR method in comparison with outranking methods, Eur. J. Oper. Res. 178 (2) (2004), 514-529.

[6] S. Opricovic, G.H. Tzeng, Multi-criteria planning of post-earthquake sustainable reconstruction, Comput.-Aided Civil Infr. Eng. 17 (3) (2002), 211-220.

[7] S. Opricovic, A fuzzy compromise solution for multicriteria problems, Int. J. Uncertain. Fuzziness Knowl.-Based Syst. 15 (3) (2007), 363-380.

[8] T.C. Wang, T.H. Chang, Fuzzy VIKOR as a resolution for multi-criteria group decision-making, In The 11th International Conference on Industrial Engineering and Engineering Management. 2005, 352-356.

[9] A. Shemshadi, H. Shirazi, M. Toreihi, M.J. Tarokh, A fuzzy VIKOR method for supplier selection based on entropy measure for objective weighting. Expert Syst. Appl. 38 (10) (2011), 12160-12167.

[10] H.-C. Liu, L. Liu, J. Wu, Material selection using an interval 2-tuple linguistic VIKOR method considering subjective and objective weights, Mater. Des. (1980-2015). 52 (2013), 158-167.

[11] M.S. Kuo, G.S. Liang, A soft computing method of performance evaluation with MCDM based on interval-valued fuzzy numbers, Appl. Soft Comput. 12 (1) (2012), 476-485.

[12] A. Sanayei, S. F. Mousavi, A. Yazdankhah, Group decision making process for supplier selection with VIKOR under fuzzy environment, Expert Syst. Appl. 37 (1) (2010), 24-30.

[13] N. Yalcin, A. Bayrakdaroglu, C. Kahraman, Application of fuzzy multi-criteria decision making methods for financial performance evaluation of Turkish manufacturing industries, Expert Syst. Appl. 39 (1) (2012), 350-364.

[14] X. Y. Zhao, S. Tang, S. L. Yang, K.D Huang, Extended VIKOR method based on cross-entropy for interval-valued intuitionistic fuzzy multiple criteria group decision making, J. Intell. Fuzzy Syst. 25 (4) (2013), 1053-1066.

[15] K. Devi, Extension of VIKOR method in intuitionistic fuzzy environment for robot selection, Expert Syst. Appl. 38 (11) (2011), 14163-14168.

[16] N. Zhang, G.W Wei, Extension of VIKOR method for decision making problem based on hesitant fuzzy set, Appl. Math. Model. 37 (7) (2013), 4938-4947.

[17] W. R. Zhang, Yin-Yang bipolar fuzzy sets, In 1998 IEEE International Conference on Fuzzy Systems Proceedings. 1998, 835-840.

[18] P.L. Yu, A class of solutions for group decision problems. Manage. Sci. 19 (8) (1973), 936-946.

[19] M. Zeleny, Multiple Criteria Decision Making, McGraw-Hill, New York, 1982. 